\title{
SUBLEVEL SETS OF CERTAIN EXTREMAL FUNCTIONS
}

\author{
NGUYEN QUANG DIEU and TANG VAN LONG
}

\section{Introduction}

Let $D$ be a domain in $C^{n}$. An upper semicontinuous function $u: D \rightarrow$ $[-\infty, \infty)$ is called plurisubharmonic if the restriction of $u$ to each complex line is subharmonic (we allow the constant function $-\infty$ to be plurisubharmonic). The set of plurisubharmonic functions on $D$ is denoted by $\mathscr{P} \mathscr{S} \mathscr{H}(D)$. Let $E$ be a subset of $D$ and $a \in D$. The relative extremal function $u_{E, D}$, the Green function $g_{a, D}$ with pole at $a$ and the global extremal (also called Siciak extremal) function $V_{E}$ of $E$ are defined as follows

$$
\begin{aligned}
u_{E, D}(z)=\sup \{v(z): & \left.v \in \mathscr{P} \mathscr{S} \mathscr{H}(D), v \leq 0,\left.v\right|_{E} \leq-1\right\} \\
g_{a, D}(z)=\sup \{v(z): & v \in \mathscr{P} \mathscr{S} \mathscr{H}(D), v<0 \\
& v(\xi)=\log |\xi-a|+O(1), \text { when } \xi \rightarrow a\}
\end{aligned}
$$

$$
V_{E}(z)=\sup \left\{v(z): v \in \mathscr{L}\left(\mathrm{C}^{n}\right),\left.v\right|_{E} \leq 0\right\}
$$

where $\mathscr{L}\left(\mathrm{C}^{n}\right)$ contains all plurisubharmonic function $u$ on $\mathrm{C}^{n}$ satisfying $u(z) \leq$ $\log ^{+}|z|+c_{u}$, where $c_{u}$ is some constant depending on $u$. These extremal functions are important objects of pluripotential theory, in particular, in connection with problems of determining capacity of sets and approximation of holomorphic functions. See [7], [13] and [16] for more details. We should remark that, in higher dimensions, it is virtually impossible to compute precisely $u_{E, D}$, $g_{a, D}$ and $V_{E}$, even for simply looking sets. Thus it may be of interest to study the geometry of the sublevel sets of these functions. Recall also that a subset $X$ of $\mathrm{C}^{n}$ is said to be convex if for every pair $z, w \in E$, the line segment connecting $z$ and $w$ also belongs to $E$. The following results are known.

Theorem 1.1 ([3], [9]). Let $D$ be an open convex domain in $\mathrm{C}^{n}$ and $E$ compact convex subset of $D$. Then for every $t \in(-1,0)$, the sublevel set $\left\{z \in D: u_{E, D}(z)<t\right\}$ is convex.

Received February 9, 2006. 
THEOREM 1.2 ([8], [10]). Let $E$ be a compact convex subset set in $C^{n}$. Then for every $t>0$, the sublevel set $\left\{z \in D: V_{E}(z)<t\right\}$ is convex.

Theorem 1.3 ([8]). Let $D$ be a convex domain in $\mathrm{C}^{n}$ and $a \in D$. Then for every $t<0$, the sublevel set $\left\{z \in D: g_{a, D}(z)<t\right\}$ is convex.

The original proofs of the above theorems are complicated and require lengthy preparation. More precisely, Theorems 1.2 and 1.3 are proved by using Lempert's powerful machinery of constructing extremal disks on convex domains, Theorem 1.1 is proved in [9] via Poletsky's theory on holomorphic disks and in [3] by considering the structure of the polynomially convex hull of sets with convex fibers over the unit circle.

The goal of this paper is to present a unified approach to the problem of determining convexity of sublevel sets for $u_{E, D}, V_{E}, g_{a, D}$. More precisely, we will give different proofs to the above theorems, which are based on the product properties for these extremal functions. This approach will also enable us to study the geometry of sublevel sets of extremal plurisubharmonic functions on complex varieties. In this more general context, the notion of convexity is replaced by a notion of connectivity. See the next section for the precise definition. The last section includes a proof of the product property for the relative extremal functions on complex varieties.

AcKNOWLedgements. This paper is an expanded version of the master thesis of the second named author. Our work is supported by the National Research Program in Natural Sciences of Viet Nam.

\section{Geometry of sublevel sets of $u_{E, D}, V_{E}, g_{a, D}$}

We start off by giving a proof of Theorem 1.3, this will enable us to see the connection between the product property of Green functions and geometry of its sublevel sets.

Proof of Theorem 1.3. Fix $z, w \in D$ such that $g_{a, D}(z)<t, g_{a, D}(w)<t$. By the product formula (see Theorem 2 in [6]) we have

$$
g_{(a, a), D \times D}(z, w)=\max \left(g_{a, D}(z), g_{a, D}(w)\right)<t .
$$

By (2) we have for all $\lambda \in(0,1)$

$$
g_{a, D}(\lambda z+(1-\lambda) w) \leq g_{(a, a), D \times D}(z, w) .
$$

It follows that $\left\{g_{a, D}<t\right\}$ is convex for all $t<0$

Before formulating a variation on Theorem 1.2, it seems appropriate to recall some elements of global extremal functions on complex varieties. Let 
$D$ be a complex subvariety of pure dimension $k$ in a domain $\Omega$ in $\mathrm{C}^{n}$ and $G$ be an open subset of $D$. An upper semicontinuous function $u$ on $G$ is called plurisubharmonic if $u \circ f$ is subharmonic on the unit disk $\Delta \subset \mathrm{C}$ for every holomorphic mapping $f: \Delta \rightarrow G$. Denote by $\mathscr{P} \mathscr{S} \mathscr{H}(G)$ the set of plurisubharmonic functions on $G$. By a deep result of Fornaess and Narasimhan in [5], $u \in \mathscr{P} \mathscr{S} \mathscr{H}(G)$ if and only if near every point $a \in G, u$ is the restriction to $G$ of a plurisubharmonic function on some neighbourhood of $a$ (in $\Omega$ ). Following W. Stoll (see [14] and [16]), we call a continuous exhaustion function $g \in \mathscr{P} \mathscr{S} \mathscr{H}(D)$ with values in $(-\infty, \infty)$ a parabolic potential if $g$ satisfies, in the sense of currents, the complex Monge-Ampère equation $\left(d d^{c} g\right)^{k}=0$ on $D \backslash g^{-1}(-\infty)$, where $d=\partial+\bar{\partial}, d^{c}=i(\bar{\partial}-\partial)$. Let $\mathscr{L}_{g}(D)$ denote the set of $u \in \mathscr{P} \mathscr{S} \mathscr{H}(D)$ such that

$$
u(z) \leq c_{u}+g^{+}(z), \quad \forall z \in D,
$$

where $g^{+}=\max (g, 0)$. Now we define for each bounded subset $E$ of $D$ the function

$$
V_{E}(z)=\sup \left\{v(z): v \in \mathscr{L}_{g}(D),\left.v\right|_{E} \leq 0\right\}, \quad \forall z \in D .
$$

In the case, $D=\Omega=\mathrm{C}^{n}$, and $g(z)=\log |z|$, we recover the global extremal function of $E$ defined in (3). For more details on these matters, the reader may consult Section 3 of [16].

We also denote by $\mathscr{A}(\Omega)$ the set of all families $\Phi:=\left\{\varphi_{\lambda}\right\}_{0 \leq \lambda \leq 1}$, where $\varphi_{\lambda}: \Omega \times \Omega \rightarrow \Omega$ is holomorphic and the function $\lambda \mapsto \varphi_{\lambda}(z, w)$ is continuous when $z, w$ are fixed. Moreover,

$$
\varphi_{0}(z, w)=z, \quad \varphi_{1}(z, w)=w, \quad \forall(z, w) \in \Omega \times \Omega .
$$

A subset $X$ of $\Omega$ is called $\Phi$-connected for some $\Phi \in \mathscr{A}(\Omega)$ if for all $\lambda \in$ $[0,1], \varphi_{\lambda}(X \times X)=X$. Again, if $D=\Omega=\mathrm{C}^{n}$ and $\varphi_{\lambda}(z, w)=\lambda z+(1-\lambda) w$, then $\Phi$-connectivity of $X$ is equivalent to its convexity.

THeOREm 2.1. Let $D$ be a locally irreducible complex subvariety of pure dimension in a domain $\Omega$ in $\mathrm{C}^{n}$, and $E$ a compact subset of $D$. Assume that there is a parabolic potential $g$ on $D$ and $\Phi \in \mathscr{A}(\Omega)$ such that the following conditions are satisfied

(a) D and E are $\Phi$-connected.

(b) $g^{+}\left(\varphi_{\lambda}(z, w)\right) \leq \max \left(g^{+}(z), g^{+}(w)\right)$ for all $(z, w, \lambda) \in D \times D \times[0,1]$. Then for all $t>0$ the set $\left\{z \in D: V_{E}(z)<t\right\}$ is $\Phi$-connected.

Proof. Set

$$
\tilde{g}(z, w)=\max (g(z), g(w)), \quad \forall(z, w) \in D \times D .
$$


By Proposition 3.4 and Example 3.5 in [16], $\tilde{g}$ is a parabolic potential for the complex subvariety $D \times D$ of $\Omega \times \Omega$. Now we fix $t>0$ and two points $z, w$ in $D$ such that $V_{E}(z)<t, V_{E}(w)<t$. Using the product formula (Theorem 4.5 in [16]) we have

$$
V_{E \times E}(z, w)=\max \left(V_{E}(z), V_{E}(w)\right)<t,
$$

where $V_{E \times E}$ is the global extremal function of $E \times E$ with respect to the potential $\tilde{g}$. Observe that for every $\lambda \in(0,1)$, by $(a)$ and $(b)$ we have

$$
V_{E}\left(\varphi_{\lambda}(z, w)\right) \leq V_{E \times E}(z, w) .
$$

It follows that $\left\{V_{E}<t\right\}$ is $\Phi$-connected for all $t>0$.

Remark. In the case where $D=C^{n}$ and $g=\log |z|$, we may apply a more elementary version of the product formula (see Proposition 5.9 in [13]) to reach the same conclusion.

Now we move to a variation of Theorem 1.1, which is in the same spirit as Theorem 2.1. At this point, some background on relative extremal functions on complex varieties is necessary. Let $D$ be a locally irreducible complex subvariety of a domain $\Omega \subset \mathrm{C}^{n}$ and $E \subset D$ then the relative extremal function $u_{E, D}$ is defined, in analogy with (1), as follows

$$
u_{E, D}(z)=\sup \left\{v(z): v \in \mathscr{P} \mathscr{S} \mathscr{H}(D), v \leq 0,\left.v\right|_{E} \leq-1\right\},
$$

We say that $E$ is regular if $u_{E, D}$ is plurisubharmonic on $D$. Moreover, $E$ is strongly regular if $E$ is regular, $u_{E, D}$ is continuous on $D$ and $\lim _{\xi \rightarrow \partial D} u_{E, D}(\xi)$ $=0$. Observe that the condition on strong regularity of $E$ implies that $D$ admits a negative continuous plurisubharmonic exhaustion function. For more background on relative extremal functions on complex varieties, the reader may consult [15].

The result below gives a sufficient condition for the regularity of a given compact set.

Proposition 2.2. Let $D$ be a locally irreducible complex subvariety of a bounded domain $\Omega$ in $\mathrm{C}^{n}$. Assume that $E$ is a compact subset of $D$ satisfying the following condition

(a) For every $a \in \partial E$ (relative to D), there exists a holomorphic map $\varphi$ : $\Delta \rightarrow \Omega$, where $\Delta$ is the open unit disk in $C$ such that $\varphi(0)=a$ and $\varphi(\Delta)$ intersects $E^{0}$, the interior of $E$ (relative to $D$ ) in a continuous curve terminating at $a$.

Then $E$ is regular. Moreover, $E$ is strongly regular if, in addition, the following conditions are fullfiled 
(b) For every point $a \in D \backslash E$, there exists $u_{a} \in \mathscr{P} \mathscr{S} \mathscr{H}(D), u_{a}<0$ such that $-\infty<\sup _{E} u_{a}<u_{a}(a)$.

(c) $\Omega$ is hyperconvex i.e., there exists a negative continuous plurisubharmonic exhaustion function on $\Omega$.

REMARKS. (i) In case $D$ is a domain in $C^{n}$, the condition (a) is nothing more than a slight improvement of the well known analytic accessibility condition due to Plesinak. See [7], p. 201.

(ii) If $K$ and $L$ are two compact sets of complex subvarieties $D_{1}$ and $D_{2}$ in domains $\Omega_{1} \subset C^{n}, \Omega_{2} \subset C^{p}$. Assume that $K$ and $L$ satisfy the condition (a) (resp. (a) and (b)) then $K \times L$ also satisfies the condition (a) (resp. (a) and (b)).

(iii) If $D$ is a hyperconvex domain in $C^{n}$ and $E$ is a compact convex subset of $D$ with $E^{0} \neq \varnothing$ then $E$ satisfies (a) and (b). Thus $E$ is strongly regular with respect to $D$.

Proof. By Theorem 1.5 in [15], the function $u_{E, D}^{*}$ is plurisubharmonic on $D$. Clearly $u_{E, D}^{*} \equiv-1$ on $E^{0}$. It follows from $(a)$ and the classical fact that every point of a continuous curve in $C$ is non thin, that $u_{E, D}^{*} \equiv-1$ on $\partial E$. Thus $u_{E, D}^{*} \equiv-1$ on $E$. Therefore $u_{E, D}$ is plurisubharmonic on $D$, so $E$ is regular. Now, assume, in addition, that conditions (b) and (c) hold. It follows from (4) that $\lim _{\xi \rightarrow \partial D} u_{E, D}(\xi)=0$. Further, by Theorem 1.7 in [15], $u_{E, D}$ is continuous on $D$. It remains to show that $u_{E, D}>-1$ outside $E$. For this, fix $a \in D \backslash E$. Choose $u_{a} \in \mathscr{P} \mathscr{S} \mathscr{H}(D), u_{a}<0$ such that $-\infty<\lambda_{a}:=\sup _{E} u_{a}<u_{a}(a)$. Set $v_{a}=\max \left(-\frac{u_{a}}{\lambda_{a}},-1\right)$. Then from (3.3) we infer $u_{E, D}(a) \geq v_{a}(a)>-1$. We are done.

Now we come to an analogue of Theorem 1.1 in the setting of complex varieties.

THEOREM 2.3. Let $D$ be a locally irreducible complex subvariety of a pseudoconvex domain $\Omega$ in $\mathrm{C}^{n}$. Let $E$ be a compact subset of $D$ satisfying conditions (a) and (b) in Proposition 2.2. Assume that $D$ and $E$ are $\Phi$-connected for some $\Phi \in \mathscr{A}(\Omega)$. Then for every $t \in(-1,0)$, the set $\left\{u_{E, D}<t\right\}$ is also $\Phi$-connected.

Proof. Fix two points $z, w \in D$ such that $u_{E, D}(z)<t, u_{E, D}(w)<t$. By applying the product formula (see Theorem 3.1 in the next section) we get

$$
u_{E \times E, D \times D}(z, w)=\max \left(u_{E, D}(z), u_{E, D}(w)\right)<t .
$$

By (3.3) we have for each $\lambda \in(0,1)$

$$
u_{E, D}\left(\varphi_{\lambda}(z, w)\right)=u_{\varphi_{\lambda}(E \times E), \varphi_{\lambda}(D \times D)}\left(\varphi_{\lambda}(z, w)\right) \leq u_{E \times E, D \times D}(z, w)<t .
$$


This implies that $\left\{u_{E, D}<t\right\}$ is $\Phi$-connected.

Remark. If $D$ is a domain in $C^{n}$ then we can apply the product formula in [4] to reach the same conclusion.

As an application of Theorem 2.3, we have

Proposition 2.4. Let $p, q$ be two norms in $\mathrm{C}^{n}$. Assume that $E:=\{q \leq 1\}$ is a compact subset of $D:=\{p<1\}$. Then for every $t \in(-1,0)$, every $\lambda \in(0,1)$ and every $k \geq 1$ we have

$$
u_{E, D}(z)<t, u_{E, D}(w)<t \Rightarrow u_{E, D}\left(\lambda^{k} z+(1-\lambda) w\right)<t .
$$

Proof. Fix $k \geq 1$. Consider the family $\Phi:=\left\{\varphi_{\lambda}\right\}_{0 \leq \lambda \leq 1}$, where

$$
\varphi_{\lambda}(z, w):=\lambda^{k} z+(1-\lambda) w, \forall(z, w, \lambda) \in C^{n} \times C^{n} \times[0,1] .
$$

Since $p, q$ are norms in $\mathrm{C}^{n}$, we can check that $\Phi \in \mathscr{A}(D)$ and $E$ is $\Phi$ connected. Notice that $E$ is convex and contains the origin, thus $E$ satisfies (a), (b), (c) of Proposition 2.2. The desired conclusion now follows from Theorem 2.3.

\section{Product property for $\boldsymbol{u}_{E, D}$}

The following result, in the case where $D_{1}, D_{2}$ are domains in complex Euclidean spaces, was proved in more general contexts in [1] and [4]. See also Theorem 1.1 in [11] for an earlier (also weaker) result.

TheOREM 3.1. Let $D_{1}, D_{2}$ be locally irreducible complex subvarieties of pure dimension in domains $\Omega_{1} \subset \mathrm{C}^{n}, \Omega_{2} \subset \mathrm{C}^{p}$ and $E_{1} \subset D_{1}, E_{2} \subset D_{2}$ be compact sets. Assume that $E_{1}$ (resp. $E_{2}$ ) satisfies condition (a) (resp. (a) and (b)) in Proposition 2.2 and $\Omega_{2}$ is pseudoconvex. Then we have

$$
u_{E_{1} \times E_{2}, D_{1} \times D_{2}}(z, w)=\max \left(u_{E_{1}, D_{1}}(z), u_{E_{2}, D_{2}}(w)\right), \quad \forall(z, w) \in D_{1} \times D_{2} .
$$

The proof that follows is strongly inspired by Theorem 2 in [6]. We need the following lemma, which is probably of independent interest. See also Lemma 5 in [6].

Lemma 3.2. Let $D$ be a locally irreducible complex subvariety of a domain $\Omega$ in $\mathrm{C}^{n}$ and $u$ be a plurisubharmonic function on $D \times \Delta$. Assume that for each $z \in D$, the subharmonic function $u(z, \cdot)$ depends only on $|\lambda|$. Then for every negative plurisubharmonic function $v$ on $D$, the function $\varphi(z)=u\left(z, e^{v(z)}\right)$ belongs to $\mathscr{P} \mathscr{S} \mathscr{H}(D)$. 
Proof. Since for each $z \in D$, the function $\lambda \mapsto u(z, \lambda)$ depends only on $|\lambda|$, we infer that this function is increasing and convex function of $\log |\lambda|$. It is then easy to check that $\varphi$ is upper semicontinuous on $D$. Now we fix $z_{0} \in D$, choose a small ball $U$ around $z_{0}$ in $\Omega$ such that $v$ is the restriction to $U \cap D$ of a plurisubharmonic $\tilde{v}$ on $U$. Let $U^{\prime}$ be another ball around $z_{0}$ which belongs compactly to $U$. Select a sequence $\left\{v_{j}\right\}_{j \geq 1}$ of negative continuous plurisubharmonic functions on neighbourhoods of $\overline{U^{\prime}}$ such that $v_{j} \downarrow v$ on $U^{\prime} \cap D$. Set

$$
\varphi_{j}(z)=u\left(z, e^{v_{j}(z)}\right), \quad \forall z \in U^{\prime} .
$$

Since $\varphi_{j} \downarrow \varphi$ on $U^{\prime} \cap D$, it is enough to show that $\varphi_{j}$ is plurisubharmonic on $U^{\prime} \cap D$. Fix $j \geq 1$, using a classical theorem of Bremermann (see Theorem 9 in [12]), we can approximate $v_{j}$ uniformly on $\overline{U^{\prime}}$ by a sequence of plurisubharmonic functions

$$
v_{j, k}=\frac{1}{m_{k}} \max \left(\log \left|f_{1, j}\right|, \ldots, \log \left|f_{m_{k}, j}\right|\right),
$$

where $f_{1, j}, \ldots, f_{m_{k}, j}$ are holomorphic functions on a $U$ and $m_{k}$ is a positive integer. Thus, it suffices to show that for every positive integer $m$, every holomorphic function $f$ on $U$ satisfying $\sup _{U^{\prime}}|f|<1$, the function

$$
\psi(z)=u\left(z,|f(z)|^{1 / m}\right)
$$

is plurisubharmonic on $U^{\prime} \cap D$. By perturbing $f$ slightly, we may assume that

$$
S:=\left\{z \in U^{\prime} \cap D: f(z)=0\right\}
$$

is nowhere dense in $D$. Notice that $\psi$ is upper semicontinuous on $U^{\prime} \cap D$. Moreover, by the assumptions made on $u$, we have $\psi$ is plurisubharmonic on $D \backslash S$. By the removable singularity theorem for plurisubharmonic functions on complex varieties (Theorem 1.7 in [2]), $\psi$ extends to a plurisubharmonic function $\tilde{\psi}$ on $U^{\prime} \cap D$. Finally, using the plurisubharmonicity of the function $z \mapsto u(z, 0)$ on $U^{\prime} \cap D$, and monotonicity of $t \mapsto u(z, t)$, for every $z^{*} \in S$ we have

$$
\begin{aligned}
\psi\left(z^{*}\right) & =\limsup _{z \rightarrow z^{*}, z \in\left(U^{\prime} \cap D\right) \backslash S} u(z, 0) \leq \limsup _{z \rightarrow z^{*}, z \in\left(U^{\prime} \cap D\right) \backslash S} u\left(z,|f(z)|^{1 / m}\right) \\
& =\tilde{\psi}\left(z^{*}\right) \leq \psi\left(z^{*}\right) .
\end{aligned}
$$

Thus $\psi=\tilde{\psi}$ on $S$. This completes the proof of the lemma.

The next lemma is presumably well known, although we have not been able to find it in the literature in this form. 
Lemma 3.3. Let $D$ be a complex subvariety of pure dimension in a domain $\Omega$ in $\mathrm{C}^{n}$. Let $u$ and $v$ be plurisubharmonic functions on D. Assume that $u \leq v$ a.e. on $D^{*}$, the smooth locus of $D$. Then $u \leq v$ everywhere on $D$.

Proof. By the theory of plurisubharmonic functions on open sets in $\mathrm{C}^{n}$ we have $u \leq v$ on $D^{*}$. Now fix a point $a \in D \backslash D^{*}$. Select a holomorphic mapping $f: \Delta \rightarrow D$ such that $f(0)=a, f(\Delta) \not \subset D \backslash D^{*}$. It follows that 0 is an isolated boundary point of $f^{-1}\left(D^{*}\right)$. Since $u \circ f$ is subharmonic on $\Delta$, we have

$$
u(a)=(u \circ f)(0)=\limsup _{t \rightarrow 0, t \neq 0}(u \circ f)(t) \leq \limsup _{t \rightarrow 0, t \neq 0}(v \circ f)(t) \leq v(a) .
$$

This completes the proof.

Proof of Theorem 3.1. The proof is divided into two steps.

Step 1. We will show that it is enough to prove (3.3) in the case where $E_{2}=\Delta^{\prime}:=\{z: \log |z| \leq-1\}$ and $D_{2}=\Delta:=\{z:|z|<1\}$. For this purpose, we assume that (3.3) holds with $E_{2}=\Delta^{\prime}$ and $D_{2}=\Delta$. Consider sequences of bounded domain $\Omega_{1}^{j} \uparrow \Omega_{1}, \Omega_{2}^{j} \uparrow \Omega_{2}$ where $\Omega_{2}^{j}$ are hyperconvex for all $j \geq 1$. It is easy to check that

$$
\begin{aligned}
& u_{E_{1}, D_{1} \cap \Omega_{1}^{j}} \downarrow u_{E_{1}, D_{1}}, u_{E_{2}, D_{2} \cap \Omega_{2}^{j}} \downarrow u_{E_{2}, D_{2}} ; \\
& u_{E_{1} \times E_{2},\left(D_{1} \times D_{2}\right) \cap\left(\Omega_{1}^{j} \times \Omega_{2}^{j}\right)} \downarrow u_{E_{1} \times E_{2}, D_{1} \times D_{2}} .
\end{aligned}
$$

Thus we may assume further that $\Omega_{1}, \Omega_{2}$ are bounded and that $\Omega_{2}$ is hyperconvex. By Proposition 2.2 and the remark following it, we have $u_{E_{1} \times E_{2}, D_{1} \times D_{2}}$ and $u_{E_{2}, D_{2}}$ are plurisubharmonic on $D_{1} \times D_{2}$ and $D_{2}$, respectively. Moreover, the latter one is a continuous negative exhaustion function for $D_{2}$ and satisfies $\left(d d^{c} u_{E_{2}, D_{2}}\right)^{k}=0$ on $D_{2} \backslash E_{2}$, where $k=\operatorname{dim} D_{2}$.

For $z \in D_{1}, \xi \in H:=\{t:-1<\operatorname{Re} t<0\}$ we set

$$
u(z, \xi)=\sup \left\{u_{E_{1} \times E_{2}, D_{1} \times D_{2}}(z, w): u_{E_{2}, D_{2}}(w)<\operatorname{Re} \xi\right\} .
$$

It follows from (4) that

$$
u_{E_{1}, D_{1}}(z) \leq u(z, \log |\lambda|), \quad \forall(z, \lambda) \in D_{1} \times\left(\Delta \backslash \Delta^{\prime}\right) .
$$

Moreover, since $u_{E_{1} \times E_{2}, D_{1} \times D_{2}}$ is plurisubharmonic on $D_{1} \times D_{2}$, by Corollary 6.10 in [2], the function $\xi \mapsto u(z, \xi)$ is continuous subharmonic on $H$ for every $z \in D_{1}$. It follows that $u$ is upper semicontinuous on $D_{1} \times H$. Next, by Theorem 6.11 in [2], we conclude that $d d^{c} u \geq 0$ on $D_{1} \times H$ in the sense of distributions. Thus $u$ is plurisubharmonic on the smooth locus of $D_{1} \times H$. So by Theorem 1.7 in [2], there is $\tilde{u} \in \mathscr{P} \mathscr{S} \mathscr{H}\left(D_{1} \times H\right)$ such that $\tilde{u} \leq u$ on $D_{1} \times H$ and 
$\tilde{u}=u$ on the smooth locus of $D_{1} \times H$. It follows that $v(z, \lambda):=\tilde{u}(z, \log |\lambda|)$ is plurisubharmonic on $D_{1} \times\left(\Delta \backslash \Delta^{\prime}\right)$ and coincides almost everywhere with $u(z, \log |\lambda|)$. Combining this with (7), by Lemma 3.3 we get

$$
u_{E_{1}, D_{1}} \leq v \quad \text { on } \quad D_{1} \times\left(\Delta \backslash \Delta^{\prime}\right) .
$$

Since $\tilde{u} \leq u$ on $D_{1} \times H$, for a given $\left(z_{0}, \lambda_{0}\right) \in D_{1} \times \partial \Delta^{\prime}$, we have

$$
\begin{aligned}
\limsup _{(z, \lambda) \rightarrow\left(z_{0}, \lambda_{0}\right)} v(z, \lambda) & \leq \limsup _{(z, \lambda) \rightarrow\left(z_{0}, \lambda_{0}\right)} u(z, \log |\lambda|) \\
& \leq \sup _{w \in E_{2}} u_{E_{1} \times E_{2}, D_{1} \times D_{2}}\left(z_{0}, w\right) \\
& \leq u_{E_{1}, D_{1}}\left(z_{0}\right) .
\end{aligned}
$$

Set

$$
\tilde{v}(z, \lambda)= \begin{cases}v(z, \lambda) & (z, \lambda) \in D_{1} \times\left(\Delta \backslash \Delta^{\prime}\right) \\ u_{E_{1}, D_{1}}(z) & (z, \lambda) \in D_{1} \times\left(\operatorname{Int} \Delta^{\prime}\right) .\end{cases}
$$

It follows from (8) and (9) that $\tilde{v} \in \mathscr{P} \mathscr{S} \mathscr{H}\left(D_{1} \times \Delta\right)$. Obviously $v \leq-1$ on $E_{1} \times \Delta^{\prime}$. So we must have $\tilde{v} \leq u_{E_{1} \times \Delta^{\prime}, D_{1} \times \Delta}$ on $D_{1} \times \Delta$. Thus there is a subset $S$ of $D_{1} \times\left(\Delta \backslash \Delta^{\prime}\right)$ of Lebesgue measure zero such that for all $(z, \lambda) \in\left(D_{1} \times\left(\Delta \backslash \Delta^{\prime}\right)\right) \backslash S$ we have

$$
u(z, \log |\lambda|) \leq u_{E_{1} \times \Delta^{\prime}, D_{1} \times \Delta}(z, \lambda) .
$$

Since for each $z$ fixed, the function $u(z, \cdot)$ is subharmonic on $H$, an easy application of Fubini theorem implies that there exists $S^{\prime} \subset D_{1}$ having Lebesgue measure 0 such that for all $(z, \lambda) \in\left(D_{1} \backslash S^{\prime}\right) \times\left(\Delta \backslash \Delta^{\prime}\right)$ we have

$$
u(z, \log |\lambda|) \leq u_{E_{1} \times \Delta^{\prime}, D_{1} \times \Delta}(z, \lambda)=\max \left(u_{E_{1}, D_{1}}(z), \log |\lambda|\right),
$$

where the last equality comes from the assumption made at the beginning of the proof. In view of (6) we then get

$$
\begin{aligned}
& u_{E_{1} \times E_{2}, D_{1} \times D_{2}}(z, w) \leq \max \left(u_{E_{1}, D_{1}}(z), u_{E_{2}, D_{2}}(w)\right), \\
& \forall(z, w) \in\left(D_{1} \backslash S^{\prime}\right) \times D_{2} .
\end{aligned}
$$

By the plurisubharmonicity of $u_{E_{1} \times E_{2}, D_{1} \times D_{2}}$ and Lemma 3.3, we deduce that (10) holds for all $(z, w) \in D_{1} \times D_{2}$. Since the reverse inequality in (10) is trivial, the desired equality (3.3) follows.

Step 2. We will show that holds with $E_{2}=\Delta^{\prime}, D_{2}=\Delta$. By Proposition 2.2 and the remark following it, the function $u_{E_{1} \times \Delta^{\prime}, D_{1} \times \Delta}$ is plurisubharmonic on $D_{1} \times \Delta$. We also deduce from that for each $z \in D_{1}$, the function $\lambda \mapsto$ 
$u_{E_{1} \times \Delta^{\prime}, D_{1} \times \Delta}(z, \lambda)$ depends only on $|\lambda|$. Thus, using Lemma 3.2 we infer that the function

$$
\omega(z):=u_{E_{1} \times \Delta^{\prime}, D_{1} \times \Delta}\left(z, e^{u_{E_{1}, D_{1}}(z)}\right)
$$

is plurisubharmonic on $D_{1}$. Clearly $\omega \leq 0$ on $D_{1}$ and $\omega \leq-1$ on $E_{1}$. It follows that $\omega \leq u_{E_{1}, D_{1}}$ on $D_{1}$. Let $z_{0} \in D_{1}$. By the maximum principle applied to the subharmonic function

$$
\lambda \mapsto u_{E_{1} \times \Delta^{\prime}, D_{1} \times \Delta}\left(z_{0}, \lambda\right)
$$

and the closed disk $\Delta_{z_{0}}:=\left\{|\lambda| \leq e^{u_{E_{1}, D_{1}}\left(z_{0}\right)}\right\}$ we obtain

$$
u_{E_{1} \times \Delta^{\prime}, D_{1} \times \Delta}\left(z_{0}, \lambda\right) \leq \max \left(u_{E_{1}, D_{1}}\left(z_{0}\right), \log |\lambda|\right), \quad \forall \lambda \in \Delta_{z_{0}} .
$$

We get the same inequality on the annulus $\Delta \backslash \Delta_{z_{0}}$, by applying on this annulus, the maximum principle to the subharmonic function

$$
\lambda \mapsto u_{E_{1} \times \Delta^{\prime}, D_{1} \times \Delta}\left(z_{0}, \lambda\right)-\log |\lambda| .
$$

Summing up, we have shown that

$$
u_{E_{1} \times \Delta^{\prime}, D_{1} \times \Delta}(z, \lambda) \leq \max \left(u_{E_{1}, D_{1}}(z), \log |\lambda|\right), \quad \forall(z, \lambda) \in D_{1} \times \Delta .
$$

Thus we are done, since the reverse inequality is obvious.

REMARK. It is of interest to see whether the method in [4] can be extended to prove, in the most general possible form, the product property for relative extremal plurisubharmonic functions on complex varieties.

\section{REFERENCES}

1. Blocki, Z., Equilibrium measure of a product subset of $\mathrm{C}^{n}$, Proc. Amer. Math. Soc. 128 (2000), 3595-3599.

2. Demailly, J. P., Measures de Monge-Ampère et caractérisation géométrique des variétés algébriques affines, Mém. Soc. Math. France 19 (1985), 1-125.

3. Duval, J., Sur la fonction extrémale plurisousharmonique relative à deux convexes, C. R. Acad. Sci. Paris 326 (1998), 433-436.

4. Edigarian, A., and Poletsky, E., Product property of the relative extremal function, Bull. Polish Acad. Sci. Math. 45(4) (1997), 331-335.

5. Fornaess, J., and Narasimhan, R., The Levi problem on complex spaces with singularities, Math. Ann. 248 (1980), 47-72.

6. Jarnicki, M., and Pflug, P., Remarks on the pluricomplex Green functions, Indiana Univ. Math. J. 44(2) (1995), 535-543.

7. Klimek, M., Pluripotential Theory, London Math. Soc. Monogr., 1991.

8. Lempert, L., La métrique de Kobayashi et la représentation des domaines sur la boule, Bull. Soc. Math. France 109 (1981), 427-474. 
9. Larusson, F., Lassere, P., and Sigurdsson, R., Convexity of sublevel sets of plurisubharmonic extremal functions, Ann. Polon. Math. 68 (1998), 267-273.

10. Momm, S., Boundary behavior of extremal plurisubharmonic functions, Acta Math. 172 (1994), 51-76.

11. Nguyen, T. V., and Siciak, J., Fonctions plurisousharmoniques extrémales et systèmes doublement orthogonaux de fonctions analytiques, Bull. Sci. Math. 115 (1991), 235-244.

12. Sibony, N., Prolongement des fonctions holomorphes bornées et métrique de Carathéodory, Invent. Math. 29 (1975), 205-230.

13. Siciak, J., Extremal plurisubharmonic functions, Ann. Polon. Math. 39 (1981), 175-211.

14. Stoll, W., Value distribution theory on parabolic spaces, Lecture Notes in Math. 600 (1977).

15. Wikstrom, F., Continuity of the relative extremal function on analytic varieties in $\mathrm{C}^{n}$, Ann. Polon. Math. 86 (2005), 219-225.

16. Zeriahi, A., Fonction de Green pluricomplexe à pôle à l'infini sur un espace de Stein parabolique et applications, Math. Scand. 69 (1991), 89-126.

DEPARTMENT OF MATHEMATICS

HANOI UNIVERSITY OF EDUCATION (DAI HOC SU PHAM HANOI)

CAU GIAY, TU LIEM

HANOI

VIETNAM

E-mail: dieu_vn@yahoo.com, tangvan.long@gmail.com 